\title{
Recommendation and Planning through Mobile Devices in Tourism Context
}

\author{
Ricardo Anacleto, Lino Figueiredo, Nuno Luz, Ana Almeida and Paulo Novais
}

\begin{abstract}
In this paper we present a mobile recommendation and planning system, named PSiS Mobile. It is designed to provide effective support during a tourist visit through context-aware information and recommendations about points of interest, exploiting tourist preferences and context. Designing a tool like this brings several challenges that must be addressed. We discuss how these challenges have been overcame, present the overall system architecture, since this mobile application extends the PSiS project website, and the mobile application architecture.
\end{abstract}

Key words: Mobile Recommendation System, Sight Information Provider, ContextAware, Client-Server Application

\section{Introduction}

Mobile systems are becoming popular in the tourism domain, specially due to the pocket size of devices. However, their computational capabilities are still limited compared to a traditional computer. Although in recent years mobile technology has evolved significantly, they still lack performance, especially in battery life, which is the biggest obstacle to mobile performance growth [11]. These limitations must be considered in the creation of any mobile application due to possible technical, ergonomic and economic implications for the user.

There are two types of tourist support applications: mobile information guides (e.g., MultiMundus [6], GeoNotes [4]) and recommendation systems (e.g., Tourism Information Provider (TIP) [5], m-ToGuide [10], CATIS [8]) [2]. The first ones pro-

Ricardo Anacleto, Lino Figueiredo, Nuno Luz and Ana Almeida

GECAD - Knowledge Engineering and Decision Support, R. Dr. António Bernardino de Almeida, 431. 4200-072 Porto, Portugal, e-mail: \{rmao, lbf, nmal, amn\} @isep.ipp.pt

Paulo Novais

Universidade do Minho, Campus of Gualtar. 4710-057 Braga, Portugal, e-mail: pjon@di.uminho.pt 
vide important services to guide the tourist during his travel, displaying points of interest (POIs) according to contextual data. The latter recommend POIs usually according to tourist profiles, allowing the planning and selection of an appropriate trip route based on a set of POIs. Although these systems can be (and should be) integrated, very few approaches integrate both [7]. To improve user-system interaction, mobile devices play an important role since they are able to capture information about the user surroundings (context-awareness) without explicit introduction of these data by the user.

Overall, some tourism support applications like m-ToGuide and Multimundus, have been subject to evaluation with not so positive results. The most complete of the analyzed systems are CATIS and TIP. However, both of them can be improved with some features like booking, augmented reality with 4D representations of sights, and a module to help handicapped people. One aspect that is also very important is the limited capabilities of mobile devices, which none of the described systems take as a main concern.

In order to suppress the limitations found in the analyzed systems, PSiS Mobile was developed. Its foundation is the PSiS (Personalized Sightseeing Tours Recommendation System), which is a tourism decision support system that is capable of recommending points of interest to a tourist according to his personal profile. Besides its recommendation capabilities, it also aids the tourist in planning trips, all through an adaptive web portal [1]. With this implementation of PSiS, tourists can go to the web portal, register and ask for a tour recommendation for a certain region. However, on the field constant assistance for the tourist is not possible since the only mean of interaction with the system is through a web portal. In that sense, a mobile application, namely PSiS Mobile, was necessary to overcome this limitation. Its main purpose is to serve as a personal companion to the tourist. After a plan for a trip is obtained using the web portal features, PSiS Mobile is able to assist the tourist on the field by complementing and updating the plan information according to his current context. Also, context-aware recommendations of sights can be given.

On the next chapter, we present PSiS Mobile and its integration with PSiS, with some detail. Afterwards, we conclude by introducing future work in order to improve PSiS.

\section{PSiS Mobile Architecture}

PSiS Mobile was developed for the Android platform and it is an occasionally connected application [3], i.e., it does not need to be permanently connected to the Internet in order to work properly. The PSiS Mobile application is divided into four main pieces: ContextService, UserInterface, Communication Manager and Mobile Database. 


\subsection{Context Service}

Since Android applications need a service to run a task in background, a Context Service was created. This service manages all the context-awareness modules. These modules are described below:

- Location Manager, is responsible for retrieving the user position using the GPS (Global Positioning System);

- Weather Service, connects to the WorldWeatherOnline web service to get weather forecasts according to the users position. The system uses it to filter outdoor POIs, i.e., if it is raining, outdoor attractions may not be recommended;

- Phone Status Manager, to get mobile device status information. This includes battery status, Internet connectivity (Wi-Fi or 3G) and GPS activity;

- DateTime Manager, deals with all date and time information;

- Planning Service, incorporates an algorithm to re-plan the original route provided by PSiS. This algorithm was implemented using decision trees;

- Tracking Service, tracks user movement and position. For example, it keeps a record of GPS coordinates and for how long the user stays in a certain POI.

\subsection{User Interface}

In Android the user interface is represented by Activities. In PSiS Mobile, each activity represents a single screen in the application, which flow, triggered by user interaction, is depicted in fig. 1 .

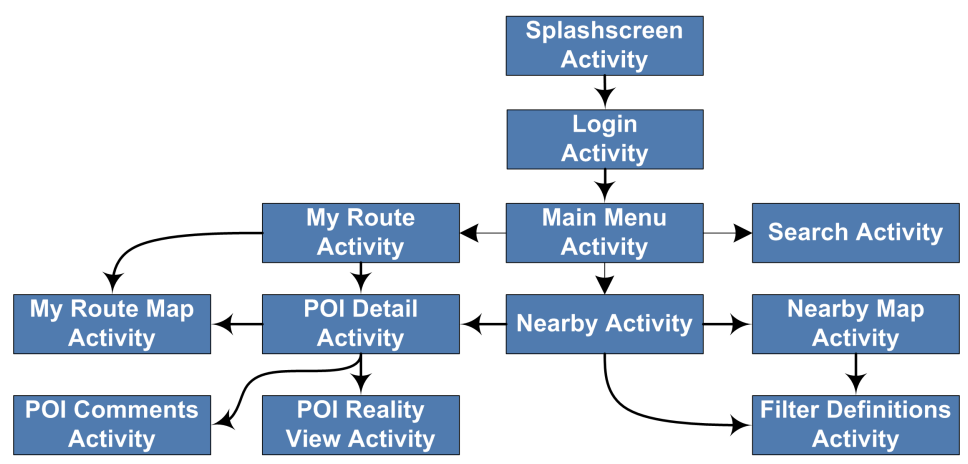

Fig. 1 Application Navigation

The Login Activity (see fig. 2(a)) is the first screen presented that demands user interaction. Here, the user has to log in by introducing an already registered user name and password. To register, he must go to the registration page in the PSiS web portal. 
After logging in, the main menu appears. This tab menu is where the user can navigate between the MyRoute Activity, the NearBy Activity and the Search Activity. During the main menu initialization, the context service is launched and begins the context retrieval.

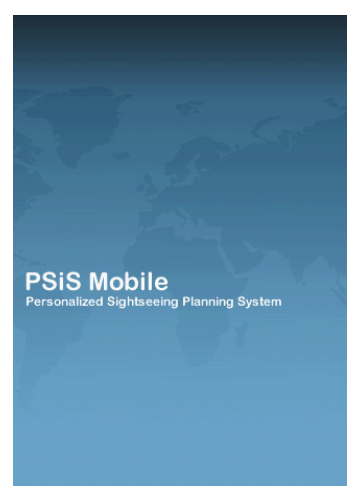

(a)

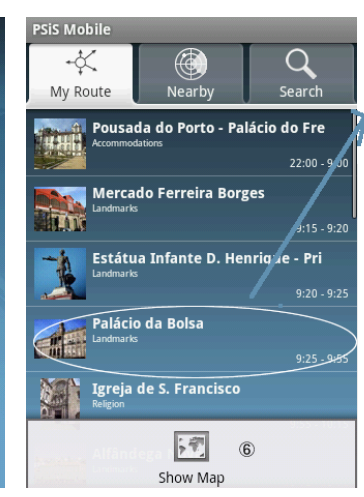

Show Map
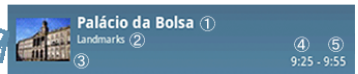

(1) - Point of interest

name;

(2) - Category Name;

(3) - Point of interest

picture;

(4) - Arrive hour;

(5) - Depart hour;

(6) - Menu to show point

of interest on map.

(b)

Fig. 2 a) SplashScreen Activity; b) MyRoute Activity

The MyRoute Activity, presented on fig. 2(b), shows the programmed route, if any, for the current day. It presents the list of POIs to visit along with their arrival and depart hours. As the tourist ends the visit to the POIs, they are excluded from the displayed route and he also can rate them. Optionally, POI detailed information is presented (in the POIDetail Activity, as shown in fig. 3) to the tourist every time he arrives to the POI.

If the tourist is behind or ahead schedule, a re-plan can be suggested by the replanning module according to the new acquired context. Additionally, by pressing the devices' "menu" button, the tourist can access the MyRouteMap Activity. This Activity is only displayed if an Internet connection is available, since it uses the Google Maps Web service to show directions from current location to the next POI.

The POIDetail Activity is launched when the tourist select a POI, and presents a simple overview of the POI without heavy and unnecessary information. Tourists can rate and input comments about the POI, which are sent to the main server, so they can be accessed through the PSiS web portal and used in future recommendations. Tourists can also access the RealityView Activity, which shows the state of things as they actually exist. Using the mobile device camera, the tourist can see the sight in the device screen and take a picture of it (see fig. 4(b)). Taken photos can be sent to the main server so they are available in the PSiS web portal. Another interesting feature is augmented reality. Using this feature, reality can be augmented by virtual computer layers. In this case, when the user is in front of a POI, more information of that sight is shown, including pictures from other users in different occasions and perspectives. To achieve this, the device must feature a camera, a compass and a GPS receiver. 


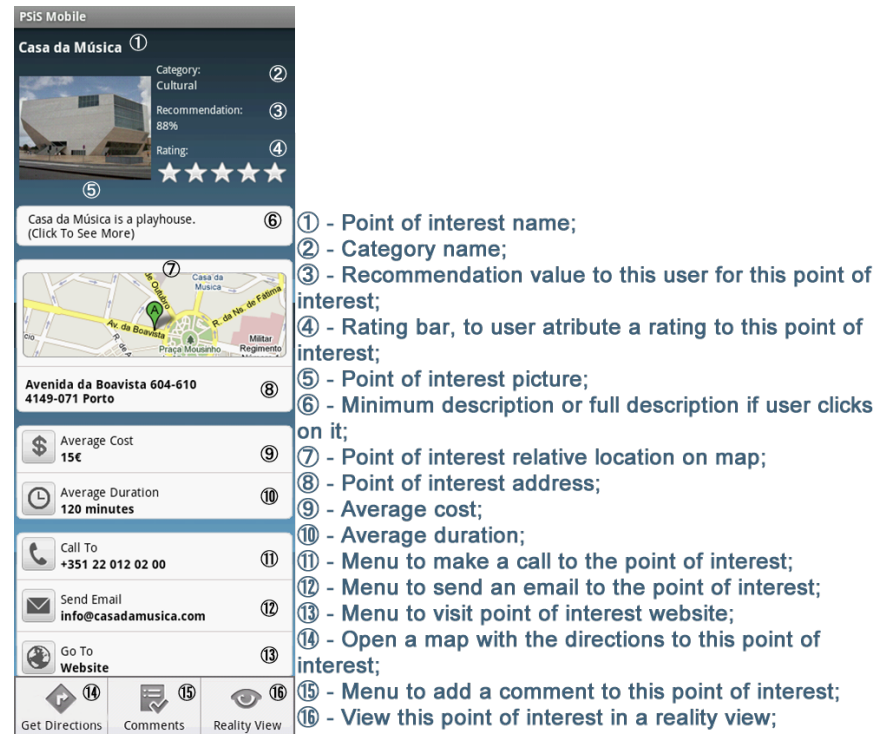

Fig. 3 PoiDetail Activity

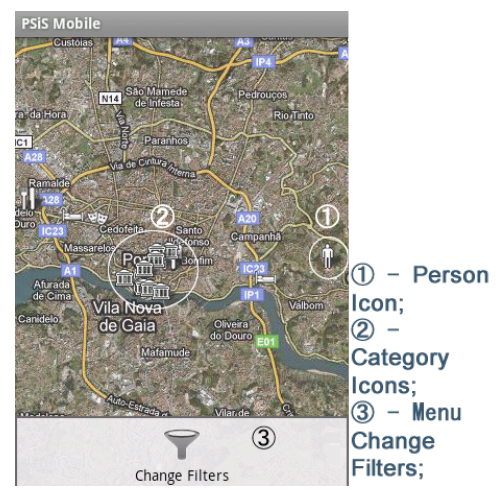

(a)

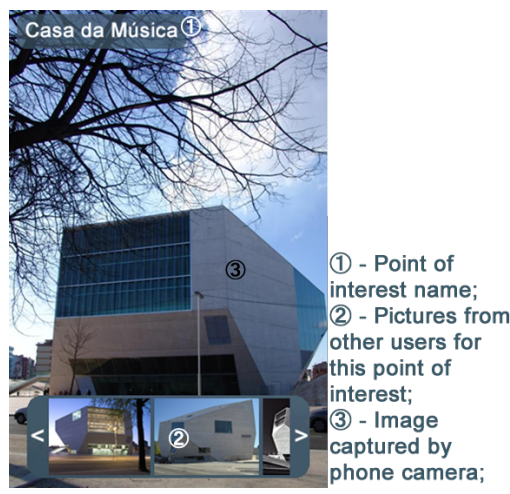

(b)

Fig. 4 a) NearbyMap Activity; b) RealityView Activity

The NearBy Activity presents nearby POIs, which are also recommended by the system. The interface is very similar to the MyRoute Activity, showing the recommendation degree and distance to the POI. The distance between tourist location and POIs is calculated using the Haversine formula [9].

The NearByMap Activity, see fig. 4(a), shows NearBy Activity information on a map, where each POI is represented by an icon. The icon corresponds to the POIs' category. For example, the restaurant category is represented by an icon with a knife and a fork. If the tourist clicks on an icon, the POI Detail Activity appears. The current user position is also presented on map through a "person" icon. 
PSiS Mobile applies simple content-based filters that rely on context-aware information. The filtering criteria are: weather condition, which filters outdoor POIs, POI schedule, which filters not available POIs according to their schedule, and current hour, which recommends restaurants instead of sights if it is lunch or dinner time. Besides these criteria, the tourist can apply additional filters using the Filter Definitions Activity. These filters can be set over:

- What categories he wants to see (e.g., Restaurants, Hotels);

- Order of the presented information in the NearBy Activity (e.g., by name, by distance, by rating);

- Maximum POI distance relative to the tourist position (from $0.1 \mathrm{Km}$ to $10 \mathrm{Km}$ );

- Amount of presented POIs: from only 1 to 30 sights.

The tourist can also search POIs using the Search Activity. This search can be performed using the POIs name, a keyword or a word present in the POI description.

\subsection{Communication Manager and Database}

Communication Manager contains the PSiS Mobile DAL, which is a Java library that provides simplified access to data stored in the local SQLite database and in the server. This layer provides an unique abstraction and management over both local and remote data sources. If there are enough local data to show to the user, it only uses the local database. Otherwise, and if an Internet connection is available, a request is made to the server. More information about client-server communications can be seen in section 3 .

\section{PSiS Middleware Architecture}

When the application is installed on the mobile device, an empty database is created. In that sense, the mobile application needs to import information from the server. This is when the middleware plays its role. First of all, when the user logs in and an Internet connection is available, the mobile device requests data to the middleware. Then, the middleware retrieves the necessary data from the server database and sends it back to the mobile device.

The middleware was developed using the Java Servlets API, and is running on a Tomcat server. To exchange information between the mobile application and the middleware, we chose the HTTP (Hypertext Transfer Protocol) protocol along with Googles' Protocol Buffers messages. This module is constituted by six submodules: Database, DAL, Authentication Service, Console, Versions Manager and Communication Manager. The database, although identical to the mobile database, includes three more fields in all tables:

- Date of when the information was sent to the mobile device; 
- User identification, to know which user has received the data;

- A Boolean field that represents the necessity or not, to update the data in the mobile device (e.g., if the user generates a new route, this field turns to true in order to indicate that it's necessary to update it in the mobile device).

For all data that is transferred to the mobile device, a copy is saved in the middleware. This way, the heavy task of controlling data versions is left to the Versions Manager sub-module instead of the mobile application.

The third middleware sub-module is a console where all the logs are presented. All requests and a responses are saved in a log table, which can be accessed using a graphical interface (a web page). A DAL is also present to create an abstraction from data present in the middleware and in the server database.

While the Authentication Service ensures data security, the Communication Manager is responsible for handling received/sent messages and redirect them to the appropriate location. Also, if the Internet connection is unstable, the system adapts itself by sending/receiving only one result at a time, instead of a list with many results. This avoids the loss of information.

In fig. 5 a sequence diagram depicts the system steps since the tourist asks for nearby POIs, until he has the result. The tourist starts by going to the NearBy Activity which requests a certain amount of POIs to the DAL. The DAL first inquires the mobile database and, if the desired amount of POIs is not available and an Internet connection is available, it passes the request to the middleware using the Protocol Buffers GetPois message. Then, the middleware sends back the requested amount of POIs using a Protocol Buffers SendPois message. If Internet connection isn't available, the application only shows the POIs present in the mobile database.

\section{Conclusion}

Up until now several tourism mobile applications were developed, but they still have several drawbacks and most of them do not account for the multiple limitations of mobile devices. In this paper, we describe the PSiS Mobile architecture, which in-

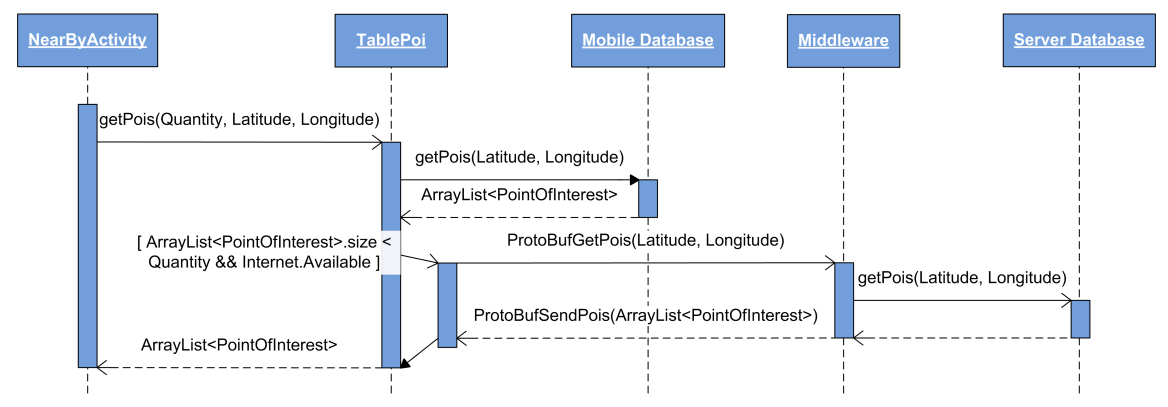

Fig. 5 Sequence Diagram Get Points of Interest 
tends to tackle these limitations. Currently, we are testing it in a real scenario in order to get realistic results to improve the system. Despite mobile device limitations, we want to provide a good user experience, giving tourists a fast and user friendly tool with context-aware adaptation, route planning, augmented reality and built-in social networking features.

In the future, we want to improve the social networking features and create algorithms to interpret the information that is recorded by the mobile application when the tourist is visiting POIs. This will be useful in order to refine even more system recommendations.

\section{Acknowledgements}

The authors would like to acknowledge FCT, FEDER, POCTI, POSI, POCI and POSC for their support to GECAD unit, and the project PSIS (PTDC/TRA/72152/2006).

\section{References}

1. Ana Almeida. Personalized sightseeing tours recommendation system. In The 13th World Multi-Conference on Systemics, Cybernetics and Informatics: WMSCI 2009, Florida, USA.

2. Ricardo Anacleto, Nuno Luz, and Lino Figueiredo. Personalized sightseeing tours support using mobile devices. In World Computer Congress 2010 (WCC2010), Brisbane, Australia.

3. Ricardo Anacleto, Nuno Luz, and Lino Figueiredo. PSiS mobile. In International Conference on Wireless Networks (ICWN2010), Las Vegas, USA, 2010.

4. F. Espinoza, P. Persson, A. Sandin, H. Nystr $\backslash$ m, E. Cacciatore, and M. Bylund. Geonotes: Social and navigational aspects of location-based information systems. In Ubicomp 2001: Ubiquitous Computing, page 217, 2001.

5. Annika Hinze and George Buchanan. Context-awareness in mobile tourist information systems: Challenges for user interaction. In International Workshop on Context in mobile HCI at the 7th International Conference on Human Computer Interaction with Mobile Devices and Services, Austria, 2005.

6. M. Kropfberger, R. Tusch, M. Jakab, J. K\pke, M. Ofner, H. Hellwagner, and L. B \sz $\backslash$ rmenyi. A multimediabased guidance system for various consumer devices. In Proceedings of the 3rd International Conference on Web Information Systems and Technologies (WEBIST07), page 8390, 2007.

7. Nuno Luz, Ricardo Anacleto, and Ana Almeida. Tourism mobile and recommendation systems - a state of the art. In International Conference on e-Learning, e-Business, Enterprise Information Systems, and e-Government (EEE2010), Las Vegas, USA, 2010.

8. Ariel Pashtan, Remy Blattler, Andi Heusser, and Peter Scheuermann. CATIS: a context-aware tourist information system. In Proceedings of IMC 2003, 4th International Workshop of Mobile Computing, Rostock, Germany, 2003.

9. C Robusto. The Cosine-Haversine formula. The American Mathematical Monthly, pages 38-40, January 1957.

10. J. Schneider and F. Schrder. The m-ToGuide Project-Development and deployment of an european mobile tourism guide. In Evolution of Broadband Services, Eurescom Summer 2003, Heidelberg, Germany, 2003.

11. R Schuler. Mobile application architecture, 2007. 